\title{
Smoker, Current Status Unknown
}

National Cancer Institute

\section{Source}

National Cancer Institute. Smoker, Current Status Unknown. NCI Thesaurus. Code

C67150.

Indicates a person who is known to have smoked but whose current smoking status is unknown. 